\title{
PAPER
}

\section{Is a combination of Tc-SPECT or perfusion weighted magnetic resonance imaging with spinal tap test helpful in the diagnosis of normal pressure hydrocephalus?}

\author{
F Hertel, C Walter, M Schmitt, M Mörsdorf, W Jammers, H P Busch, M Bettag
}

J Neurol Neurosurg Psychiatry 2003;74:479-484

See end of article for authors' affiliations

Correspondence to Dr F Hertel, Neurochirurgie im Brüderkrankenhaus, Nordallee 1, D-54292 Trier, Germany; F.Hertel@ bk-trier.de

Received 30 September 2002

Accepted in revised form 27 December 2002

\begin{abstract}
Objective: The aim of this study was to evaluate the combination of spinal tap test (STT) with cerebral perfusion measurement assessed either by Tc-bicisate-SPECT (Tc-SPECT) or perfusion weighted MRI (pwMRI), or both, for a better preoperative selection of promising candidates for shunt operations in suspected idiopathic normal pressure hydrocephalus.

Methods: 27 consecutive patients were examined with a standard clinical protocol (assessed by the Homburg Hydrocephalus Scale (HHS)) as well as with $99 \mathrm{~m}$ Tc-bicisate-SPECT ( $\mathrm{n}=27$ ) or additionally by pwMRI $(n=12)$ before and after STT. The results of these examinations were compared preoperatively for each patient and correlated with postoperative clinical outcome after shunt surgery.

Results: Nine patients showed both, a clinical improvement, and increased cerebral perfusion after STT. They underwent shunt surgery with good to excellent results. In another nine patients increasing cerebral perfusion was detected although they did not show a clear clinical improvement after STT. Six of them also received a shunt operation with good to excellent outcome. Three patients of the last group could have an operation. Nine patients did not show any clinical improvement or any kind of increasing cerebral perfusion after STT. Therefore, they did not undergo surgery. The results of SPECT and pwMRI correlated in $92 \%$ of the patients (11 of 12).

Conclusion: It is concluded that a combination of clinical assessment with SPECT or pwMRI is helpful in the preoperative selection of patients for shunting procedures with suspected NPH syndrome. This combination is a minimal invasive and objective test modality that is superior to STT alone. Further studies are necessary for a comparison of the described imaging techniques with different diagnostic tests in this difficult field of cerebral disease.
\end{abstract}

$\mathrm{T}$ he idiopathic normal pressure hydrocephalus (NPH) was first described by Adams and Hakim. ${ }^{1}$ It is characterised by clinical presentation with gait disturbance, mnestic deficit, urinary incontinence, sometimes in combination with headache and dizziness. ${ }^{2-3}$ The differentiation of brain atrophy, Parkinsons disease, vascular encephalopathy, Alzheimer's disease, or Binswanger's disease to NPH can be difficult if only morphological criteria are used, for example, enlarged ventricles. ${ }^{4-6}$ Therefore, the outcome after shunt surgery varies considerably among different series and depends upon the preoperative patient selection. ${ }^{6-8}$ The diagnostic procedures so far consisted of morphological imaging modalities such as magnetic resonance imaging (MRI), ${ }^{9}$ computed tomography $(\mathrm{CT}),{ }^{10}$ as well as clinical assessment before and after spinal tap test (STT), ${ }^{11}$ lumbar drainage, ${ }^{12}$ lumbar infusion tests, ${ }^{13}$ and prolonged continuous intracerebral pressure measurements. ${ }^{14}$

Although there is no consensus about the preoperative patient selection and diagnostic confirmation of NPH so far, clinical examination before and after lumbar STT is most widely used at present. ${ }^{711}$ The clinical assessment consists usually of a standard walking protocol, psychometric testing — such as mini-mental state test (MMS) - and incontinence protocol. ${ }^{6}{ }^{15}$ The clinical results after STT are sometimes difficult to interpret and are partially investigator dependent. There may be sometimes false negative results, because in a certain number of patients the clinical assessment is ambiguous. $^{715}$

Since as early as 1969 it was proposed that the NPH syndrome is caused by a decreased cerebral perfusion. ${ }^{16}$ Different methods of cerebral perfusion imaging such as cerebral angiography, ${ }^{16}$ Xenon-CT, ${ }^{17-19}{ }^{99 \mathrm{~m}}$ Tc-HMPAO SPECT, ${ }^{20}$ (123 I) IMP-SPECT ${ }^{21}$ showed a positive correlation between increased cerebral blood flow (assessed by different imaging modalities) and clinical improvement after CSF shunting procedures.

Perfusion weighted magnetic resonance imaging (pwMRI) is a comparatively new imaging modality that offers a measurement of changes in cerebral perfusion.

The purpose of this study was to combine the image data provided by SPECT or pwMRI, or both, with the clinical results before and after STT. The question was whether the additional perfusion data can help to improve the selection of promising candidates for successful shunting procedures in suspected NPH syndrome.

\section{METHODS}

\section{Patient population}

Between 1998 and 2000, 29 patients with suspected idiopathic $\mathrm{NPH}$ syndrome were included in the prospective study. Two patients had to be excluded from the study because the imaging examinations could not be performed due to incompliance (severe movement artefacts occurred in these patients). The remaining 27 patients, 15 men and 12 women with a mean age of 69.2 years (range 39-82 years) were evaluated The patients gave their informed consent to participate in the study.

Abbreviations: $\mathrm{NPH}$, normal pressure hydrocephalus; MRI, magnetic resonance imaging; $\mathrm{CT}$, computed tomography; $\mathrm{STT}$, spinal tap test; PwMRI, perfusion weighted magnetic resonance imaging; HHS, Homburg hydrocephalus scale; IM, index map; MTT, mean transit time; $N I$, negative integral; SPECT, ${ }^{99 \mathrm{~m}}$ Tc-bicisate single photon emmision tomography; TTP, time to peak. 


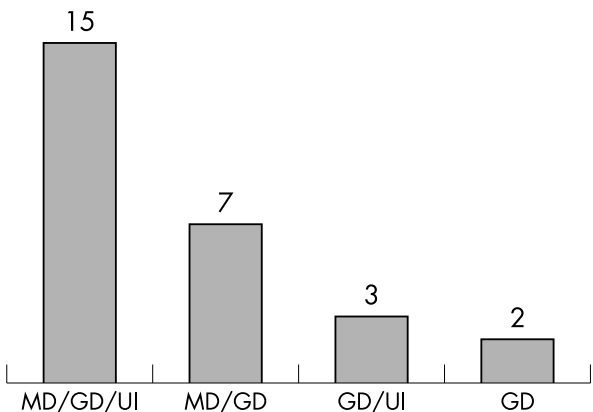

Figure 1 Symptoms of the patients. All patients 100\% suffered from GD (gait disturbance), $89 \%$ suffered from MD (mnestic deficit), $69 \%$ showed different degrees of urinary incontinence.

\section{Inclusion criteria}

All patients were suffering from one of the following symptoms of NPH syndrome or a combination of them: gait disturbance $(100 \%)$, mnestic deficit $(84 \%)$, urinary incontinence $(69 \%)$. Figure 1 shows the different combinations of clinical symptoms that led to the inclusion of patients in this study. Most of patients were suffering from the classic clinical trias-the so called Hakim trias. ${ }^{1}$ Some $33 \%$ of the patients showed additionally further symptoms of chronic hydrocephalus such as dizziness and headache. The duration of symptoms ranged from three months to two years with a mean duration of one year. In all patients a previous CT or MRI scan, or both, showed communicating hydrocephalus with an extended ventricular system. In the evaluated patients no further major cerebral abnormality was found except periventricular lucency and different degrees of deep white matter hyperintensities as a sign of vascular white matter lesions.

\section{Clinical examination}

All patients were examined by the same investigator before and after STT. The clinical examination protocol consisted of a standardised neurological examination, a walking test, a psychometric examination (MMS) and the Homburg Hydrocephalus scale (HHS), ${ }^{4}$ as well as an incontinence protocol. For the walking test the patient had to navigate a flat route of 20 metres of length three times. The middle pulse length and the required steps were measured.

Additionally, the patients were clinically assessed according to the HHS. The five symptoms were tested as previously described $^{4}$ : memory deficit and intellectual impairment; gait disturbance; urinary and bowel incontinency; headache; and dizziness. All examinations were performed before and after STT. A decrease of at least four points on the HHS scale after STT was rated as a clear clinical improvement (STT positive), a change of two or three points was rated as a questionable result (STT questionable). If the score changed less than two points no clinical improvement was assessed (STT negative). In addition to the routine follow up examinations, information was also obtained from relatives and care givers.

The clinical outcome after shunt operation was evaluated according to the Black rating scale for shunt assessment. ${ }^{22}$ This scale includes six categories (excellent, good, fair, transient, poor, or dead).

\section{Examination protocol}

All 27 patients were examined by a combination of clinical assessment before and after STT, either with ${ }^{99 \mathrm{~m}}$ Tc-bicisateSPECT only $(n=15)$, or with a combination of ${ }^{99 m}$ Tc-bicisateSPECT and pwMRI $(n=12)$.

At days 1 and 3 all patients were examined by clinical assessment and Tc-bicisate-SPECT. Twelve patients were additionally examined with pwMRI on both days.

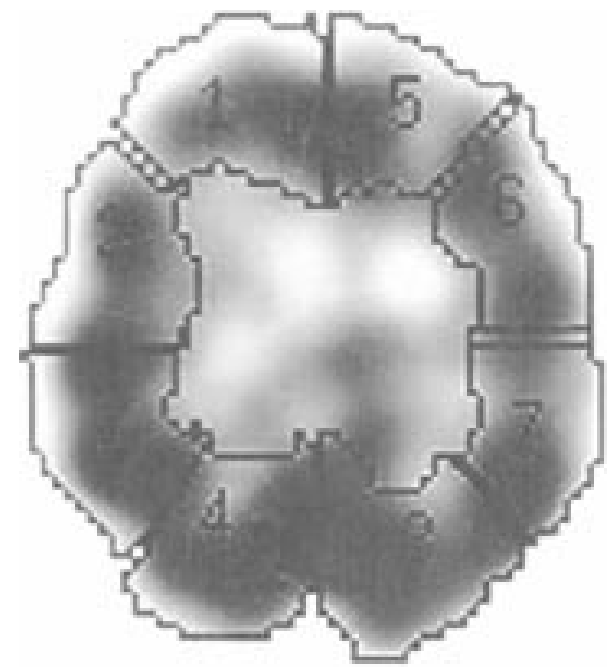

Figure 2 Tc-bicisate SPECT of a patient with suspected NPH syndrome. The regions of interest are shown.

On the the second day, at least $40 \mathrm{cc}$ of CSF were released by lumbar puncture and CSF pressure was measured. All patients had CSF pressure levels below $140 \mathrm{~mm} \mathrm{H}_{2} \mathrm{O}\left(80-130 \mathrm{~mm} \mathrm{H}_{2} \mathrm{O}\right)$.

\section{Imaging modalities \\ SPECT}

For the SPECT scan, $740 \mathrm{MBq}$ 99mTc-bicisate (Dupont) were administered intravenously after blocking of the thyroid gland by Irenat. (Bicisate is an equivalent substance to HMPAO as an agent for demonstrating cerebral blood flow). The radiotracer was injected in a silent room with the patients eyes closed. After four hours, the patients were scanned with a Toshiba single head gammacamera $(128 \times 128$ matrix $)$ in $6^{\circ}$ steps every 30 seconds in 60 projections (full circle). Reconstructions were done in horizontal, sagittal, and coronal direction after filtered backprojection with a Butterworth filter (9BW 8.0.11). The transversal slices of the examinations were matched and identical. Eight regions of interest (ROIs) were evaluated (counts per pixel) in five reconstructed slices (fig 2). The count density was normalised to the cerebellum and a ratio was calculated before/after STT. $^{20}$. An global increase of tracer consumption of at least $20 \%$ in more than half of the ROIs was evaluated as an improvement. ${ }^{20}$

\section{pwMRI}

Routine T1 and T2 weighted MR sequences were performed to image morphology of the brain on a standard $1.5 \mathrm{~T}$ clinical scanner (Gyroscan ACS-NT, Philips Medical Systems, Best, Netherlands) with a standard quadrature head coil. A susceptibility based MR perfusion sequence was performed as a 3D segmented EPI technique with the readout shifted to the next TR, resulting in TE > TR (PRESTO, TR: 19.8 ms, minimum TE: 9.5 ms, actual TE: 29 ms, flip angle $8^{\circ}$ ) as described previously. ${ }^{23}$ Twenty five slices covering the entire brain (matrix size: $64 \times 64$ ) were acquired with a time resolution of 1.9 seconds. The total scan time was 1:17 minutes to acquire 40 dynamic scans. A bolus of gadodiamid $(0.5 \mathrm{mmol} / \mathrm{ml}, 0.2$ $\mathrm{mmol} / \mathrm{kg}$ bw) (Omniscan, Nycomed, Norway) was administered with an injection rate of $8 \mathrm{ml}$ per second followed by a flush of $20 \mathrm{ml}$ saline.

\section{Data processing and image analysis of pwMRI}

All perfusion data were transferred to a workstation (Easy Vision 4.2, Philips Medical Systems, Best, Netherlands) and processed with commercially available software. Perfusion parameter maps were calculated using segmentation analysis (negative integral (NI), index map (IM), time to peak (TTP), 

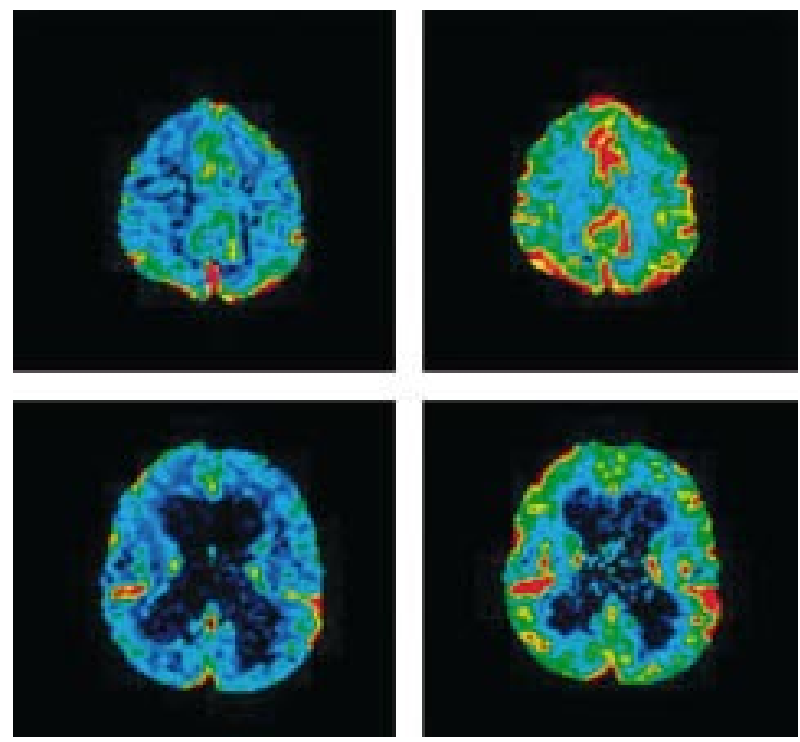

Figure 3 Perfusion weighted MRI in a patient with increasing rCBV through spinal tap test. Before (left) and after (right) spinal tap test. This patient was shunted with an excellent result.

time of arrival (TOA), median transit time (MTT)). ROIs were additionally defined by an operator. These curves were computed from the measured signal intensity changes and fitted to a gamma-variate function to correct for recirculation. Perfusion parameter maps were computed on a voxel by voxel basis comparable to other previously described techniques. The so called NI represents the area under the fitted curve. NI corresponded to the regional cerebral blood volume (rCBV). The IM that corresponded to $\mathrm{rCBF}$ was calculated as the ratio of NI and MTT.

The parameter maps (NI, IM, MTT) with identical scale and ROI measurements before and after STT were compared. The scale of each parameter was adjusted individually in the first examination to visualise significant parameter changes and was retained for the following examination. For the NI map and the IM the scale was selected such that the change from the blue to the green colour represents at least doubling of the value of the rCBV and $\mathrm{rCBF}$. The colour scale of the time curves (MTT, T0, TTP) was determined to show differences from two to five seconds. The perfusion changes were visually analysed and quantitatively evaluated by ROI analysis. ROI measurements were performed on both sides in the cerebellum and the periventricular white matter and cortex. Then mean values of the cerebellum and periventricular region were computed from these data. The perfusion data were analysed semiquantitatively and an improvement was rated visually in consensus by two neuroradiologists. They were blind to the clinical results.

\section{Surgery}

The patients who improved after STT either clinically, or by increasing perfusion in SPECT or pwMRI, respectively, were selected for surgery. These patients received ventriculoperitoneal shunts (Miethke Dual-Switch valves 10/30, 10/40). The first number of the dual switch valve determines the opening pressure in an horizontal, the second one in the upright patient position. ${ }^{24}$

Follow up examinations were performed at least three months after surgery.

\section{Statistical analysis}

To assess the relation of clinical changes (improved, not improved) and perfusion changes (improved, not improved) Fisher's exact test was performed. (SPSS 9.0.1). ${ }^{25}$
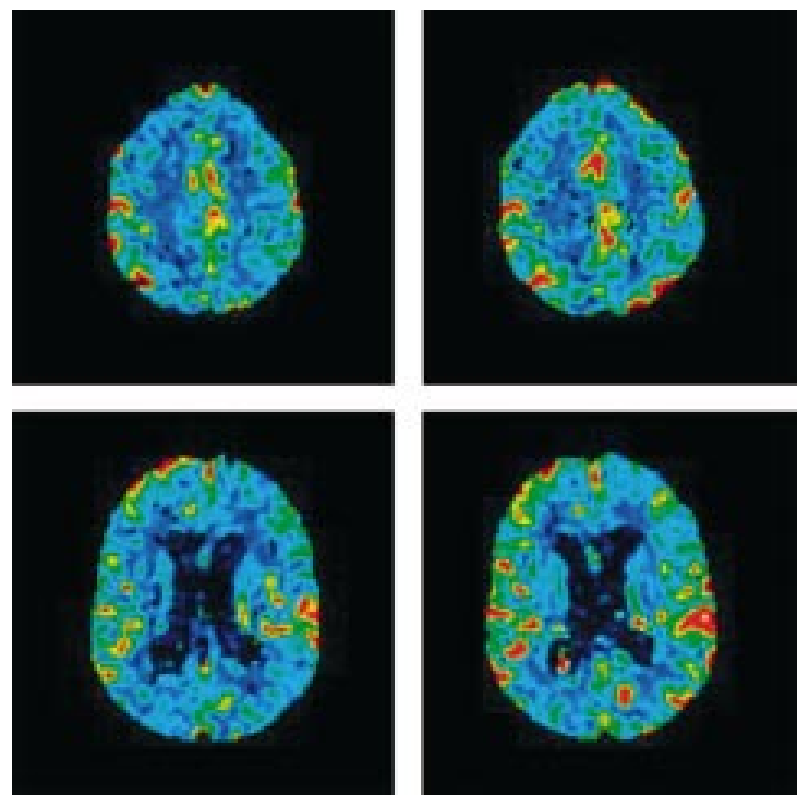

Figure 4 Perfusion weighted MRI before (left) and after spinal tap test (right). There is no significant change in $\mathrm{rCBV}$ through spinal tap test.

\section{RESULTS}

\section{Clinical examination}

Nine of the 27 patients showed a marked clinical improvement after STT in the HHS. In the remaining 18 patients the clinical examination before and after STT was rated without any noticeable change.

There was no significant difference in both groups between the HHS scores before STT (7.94 in the positive group compared with 7.22 in the negative group).

The mean CSF pressure values before STT was $100 \mathrm{~mm} \mathrm{H}_{2} \mathrm{O}$ (80-140 $\mathrm{mm} \mathrm{H}_{2} \mathrm{O}$ ). There was also no difference in CSF pressure values between the positive and the negative group.

\section{SPECT and pwMRI examinations}

Eighteen of the examined 27 patients were rated as improved according to the imaging techniques.

In those patients who were rated as imaging positive, a global increase of cerebral blood flow was seen in SPECT and of cerebral blood volume in pwMRI (fig 3). These differences were seen in the CBF in SPECT, as well as in the NI as a parameter for cerebral blood volume in pwMRI. Visually, we did not notice any differences in other parameters of pwMRI (IM, MTT, TTP). In the other nine patients no perfusion changes were detected (fig 4). (Figures 3 and 4 show examples of perfusion weighted MRI (negative integral corresponding to the relative cerebral blood volume, rCBV). In these figures, a change between two colours (blue to green, green to yellow, yellow to red) corresponds to a doubling of rCBV.)

No visible differences were found between subcortical and cortical improvement in pwMRI. A clear distinction between white and grey matter was not possible in the SPECT scans (fig 2).

The results of SPECT and pwMRI correlated in all but one patient ( 11 of 12). This patient showed a clear clinical response to STT and pwMRI was positive, but the results in SPECT did not change in any way after the STT. Remarkably, this patient had another STT in another clinic three weeks before our examination.

During the study, we did not see any negative side effects caused by ${ }^{99 m}$ Tc-bicisate-SPECT or pwMRI, respectively.

Because we had to use a single head gammacamera for the SPECT investigations, we had a scanning time of at least 30 


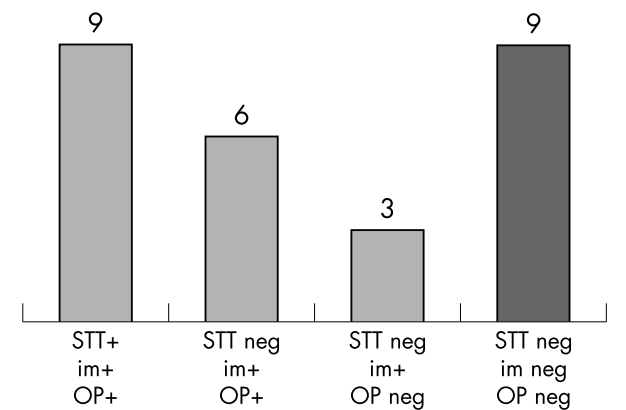

Figure 5 This figure shows the different groups according to the results of imaging and STT. Group 1 (35\%): clinical and imaging results were positive after STT, all patients were operated. Group $2 a$ $(23 \%)$ : imaging was positive. Although there was no clear clinical effect after STT, the patients were operated with good results. Group $2 \mathrm{~b}(12 \%)$ : in this group imaging was also positive, but the patients were not operated because they refused the operation $(n=2)$ or it was not possible $(n=1)$ because of some other medical reasons. In group $3(31 \%)$ neither the imaging, nor the clinical results showed any improvement after STT.

minutes, compared with the scanning time in MRI of around 20 minutes for all sequences and just around two minutes additional time for the perfusion weighted sequences.

\section{Relations of perfusion imaging and clinical examinations}

Nine of the 27 patients showed a clear clinical improvement, and a positive result in the imaging modalities (group 1) (fig 5). Another nine patients were assessed as negative or questionable according to HHS before and after STT, whereas the imaging data suggested a perfusion improvement (group $2 \mathrm{a}$ and b) (fig 5). The last nine patients did not show any clinical improvement after STT, or any positive results in imaging modalities (group 3) (fig 5) The statistical analysis showed a highly significant association between the clinical assessment and the perfusion changes $(p<0.01, w=0.5)$.

\section{Results of shunting}

All nine patients who were rated positive in imaging and clinical assessment (group 1) received a ventriculoperitoneal shunt as described above. In two patients the result was excellent, four showed a good, two a moderate, and one patient at least a temporary good result according to the Black rating scale for shunt assessment, ${ }^{22}$ which was evaluated three months postoperatively (fig 6). In the last patient, the shunt had to be removed six weeks after the operation because of a shunt related infection.

Of the second group (imaging positive, STT negative or questionable), six of nine patients received a shunt operation (group 2a). One showed an excellent, three a good, one a moderate, and one only a temporary clinical improvement according to the evaluation three months after the operation (fig 6). This last patient died from an intestinal infarction, eight weeks after the shunt operation. This was not related to the operation.

The remaining three patients of this group (group $2 \mathrm{~b}$ ) did not receive a shunt, because two patients refused the operation, and one patient suffered from a deep venous thrombosis with consecutive multiple lung embolisms. She had to be treated permanently with cumarins and therefore the risk for a shunt operation was to high.

Additionally to the above mentioned shunt infection, we had no further operation related complications. Especially, there were no clinical or radiological signs of overdrainage. The mean hospitalisation was seven days postoperatively (5-14 days).

As to be expected, after implantation of dual switch valves, no decrease of the enlarged ventricles was seen in CT/MRI after the shunt operation. ${ }^{24}$

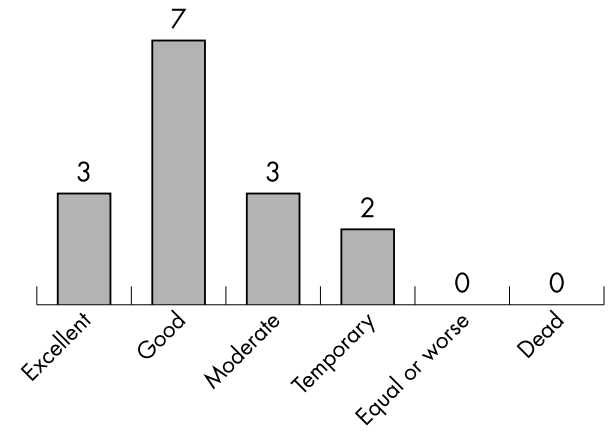

Figure 6 The clinical results of the patients after a shunt operation according to the Black rating scale for shunt assessment. Excellent, equivalent to the premorbide state. Good, slight difficulties in the activities of daily living. Moderate, gradual decrease of the symptoms. Temporary, temporary decrease of the symptoms. No change or worse, no change or worse than before the operation.

None of the patients of the third group underwent surgery, because there was no preoperative evidence for possible improvement after shunt operation.

\section{DISCUSSION}

It has become evident that a careful preoperative selection of patients with clinical suspected idiopathic NPH syndrome is crucial for the outcome in CSF shunting procedures. ${ }^{6-8} 22$

Since the first description of NPH Adams and Hakim in $1965,{ }^{1}$ a number of tests and diagnostic procedures for a proper selection of promising candidates for shunting procedures have been described. However, it is difficult to distinguish NPH from brain atrophy, using morphological imaging as CT or MRI alone, because with both methods, ventricular enlargement can be seen. ${ }^{4-8}$

In this paper, we describe a combination of a clinical test and functional imaging modalities in the selection of promising candidates for shunting procedures in patients with suspected idiopathic NPH syndrome.

Our data suggest that perfusion measurement (independent of the imaging method) is a positive predictor of shunt responsiveness in NPH syndrome.

According to different authors, the symptoms in NPH are caused by diminished cerebral perfusion in relation to an imbalance of CSF production and resorption, ${ }^{16}{ }^{19-21}$ which might be one of the pathophysiological mechanisms in the NPH syndrome.

The detection of changes in cerebral perfusion after release of CSF (as detected here by SPECT or pwMRI, or both) also shows the capacity of cerebral blood flow improvement.

In the literature, the results of cerebral perfusion measurements in NPH with different methods (for example SPECT, Xe-CT, angiography) are partially inconsistent. So far, most of the studies dealing with cerebral perfusion imaging in NPH compared perfusion parameters before and after CSFshunting procedures. ${ }^{192026}$ In most of the patients a noticeable clinical improvement postoperatively correlated positively with increasing cerebral perfusion. ${ }^{20} 28$ However, in other patients groups such as patients with neurodegenerative disorders, the reverse occurred after CSF removal- that is, a decrease in cerebral blood flow after surgery. ${ }^{18} 28$ Possible mechanisms for decreased CBF might be stretching and compression of intraparenchymatous capillaries due to a disbalance of CSF production and resorption, and therefore enlarged ventricular system. Sympathetic influences such as reflectory constriction of the vessels are also discussed, as well as stretching of the cholinergic projection fibres of the basal nucleus with consecutive impairment of the autoregulatory capacity of $\mathrm{CBF}^{17}{ }^{29}$ 
So far, only a few studies examined the effect of temporary CSF release on cerebral perfusion..$^{17}{ }^{20-32}$ In three of these reports, $\mathrm{CBF}$-measured by Xe CT-increased significantly after temporary CSF release. ${ }^{17}{ }^{21}{ }^{31}$ Only in two of these studies, $\mathrm{CBF}$ measurement was used as a predictor for the outcome after shunt surgery. ${ }^{17}{ }^{21}$ Increased CBF after temporary CSF release correlated positively with the clinical outcome after shunt surgery. ${ }^{17} 21$

However, the studies of Kristensen et al and Kushner et al failed to show an increasing CBF according to temporary CSF release..$^{26} 30$

The reasons for the negative results in their studies might be attributable to differences in technical details, as well as in the timing of their measurements. Kristensen $e t a^{26}$ injected only a dose of $550 \mathrm{mBq}$ Tc (compared with $740 \mathrm{mBq}$ in our study), which might have been resulted in a decreased signal to noise ratio. In the second study, Kushner et al ${ }^{30}$ performed their CBF measurements within one hour after CSF release. Another reason for the different results of Kushner et al might be attributable to the data analysis of the different patients groups. In their paper the perfusion data of the patients with idiopathic NPH were analysed altogether without differentiation of a responder and non-responder group. So, the mean value of perfusion measurements was given for all NPH patients. In contrast we observed two different groups of perfusion patterns (improved compared with not improved). Therefore, the mean value in the referred study might hide the individual changes of perfusion.

Though SPECT and pwMRI are semi-quantitative methods, they bear much more objectivity than clinical testing before and after STT alone. ${ }^{811} 32$ Perfusion sensitive imaging alone without the use of STT test is not able to select proper candidates for shunt operations, and does not allow to differentiate NPH from other disorders such as Alzheimer's disease. ${ }^{33}$ However, the combination of perfusion sensitive imaging (as used in this study) with a STT offers much more information, especially about the potential of perfusion improvement.

Other tests, as lumbar drainage, long time pressure wave measurement, or lumbar and ventricular infusion tests show much better results than STT only, but they are invasive and not comfortable for the patient. ${ }^{12-14}$ Especially the different kinds of infusion tests (though they show excellent results) are invasive and time intensive. ${ }^{815}$ Thus, they are limited to a few patients and can only be applied in specialised centres.

In this study, we could show that the above mentioned combination of a clinical STT with perfusion weighted imaging (SPECT/pwMRI) is safe. It is more sensitive than the clinical assessment before and after STT.

As shown by our data, $33 \%$ of the examined patients showed improved perfusion without clinical improvement after STT. Thus, the selection of promising candidates for shunt surgery was obviously improved.

There exists a limitation in this technique: patients with severe dementia who are not cooperative might not be scanned by SPECT or MRI, because of movement artefacts ( $\mathrm{n}=2$ in our study).

Comparing SPECT with pwMRI, there is a strong advantage with pwMRI: the correlation of anatomical details is much better in MRI than in SPECT. Most patients with typical NPH symptoms get an MRI scan at least once during their patient history. The pwMRI takes just around two minutes of additional scanning time. Furthermore, MRI allows to combine different imaging modalities (CSF dynamics, conventional MRI, pwMRI, primary sagittal, coronal and axial scanning). Therefore MRI might be more informative than SPECT scanning alone. We could not detect real differences in the sensitivity between both modalities with regard to cerebral perfusion changes.

In conclusion, a combination of perfusion sensitive imaging provided by SPECT or pwMRI and clinical assessment, before and after STT, is helpful, to select candidates for shunt opera- tions in patients with suspected idiopathic NPH syndrome. It is more sensitive than clinical testing before and after STT only. Though it has not been compared with a variety of other procedures in NPH diagnosis (such as long time cerebral pressure measurement, lumbar drainage, different kinds of infusion tests), so far this method is a new, promising and minimal invasive diagnostic test.

The method described offered comparable, objective measurements before and after STT in all patients. Perfusion imaging contributes to the selection of patients who may benefit from shunt procedure.

\section{ACKNOWLEDGEMENT}

We thank Professor O Kempski (Institute of Neurosurgical Pathophysiology University of Mainz) for his critical comments and his support of our work and Rene Westernhausen (Center for Neuropsychological Research) for help with the statistical analysis.

\section{Authors' affiliations}

F Hertel, M Bettag, Department of Neurosurgery, Brüderkrankenhaus Trier, Germany

C Walter, M Mörsdorf, Busch, Department of Radiology and Neuroradiology, Brüderkrankenhaus Trier

M Schmitt, W Jammers, Department of Nuclear Medicine,

Brüderkrankenhaus Trier

C Walter, Centre for Neuropsychological Research, University of Trier

Competing interests: none declared.

\section{REFERENCES}

1 Adams RD, Fischer CM, Hakim S, et al. Symptomatic occult hydrocephalus with normal cerebrospinal fluid pressure. N Engl J Med 1965;273:17-26

2 Gallassi R, Moreale A, Montagna P, et al. Binswanger's disease and normal pressure hydrocephalus. Arch Neurol 1991;48: 1156-9.

3 Krauss JK, Regel JP, Vach W, et al. Vascular risk factors and arteriosclerotic disease in idiopathic normal pressure hydrocephalusof the elderly. Stroke 1996;27:24-9.

4 Kiefer M, Steudel WI. Moderne Diagnostik und Therapie des Hydrocephalus beim älteren Menschen. Saarländisches Ärzteblatt 1994; 10:498-504.

5 Vanneste JA. Diagnosis and management of normal-pressure hydrocephalus. J Neurol 2000;247:5-14.

6 Boon AJ, Tans JT, Delwel EJ, et al. The Dutch normal pressure hydrocephalus study. Surg Neurol 2000;53:201-7.

7 Vanneste J. Three decades of normal pressure hydrocephalus: Are we wiser now? J Neurol Neurosurg Psychiatry 1994;57:1021-5.

8 Savolainen S, Hurskainen H, Paljarvi L, et al. Five-year outcome of normal pressure hydrocephalus with or without a shunt: predictive value of the clinical signs, neuropsychological evaluation and infusion test. Acta Neurochir (Wien) 2002;144:515-23.

9 Kitagaki $\mathrm{H}$, Mori $\mathrm{E}$, Ishii $\mathrm{K}$, et al. CSF spaces in idiopathic normal pressure hydrocephalus: morphology and volumetry. ANNR Am J Neuroradiol 1998;9:1277-84

10 Bradley WG Jr. Diagnostic tools in hydrocephalus. Neurosurg Clin N Am 2001;12:661-84.

11 Wickelsö CH, Andersson H, Blomstrand C, et al. Normal pressure hydrocephalus: predictive value of the cerebrospinal fluid tap test. Acta Neurol Scand 1986;73:566-73

12 Walchenbach R, Geiger E, Thomeer RT, et al.The value of temporary external lumbar CSF drainage in predicting the outcome of shunting on normal pressure hydrocephalus. J Neurol Neurosurg Psychiatry 2002;72:503-6.

13 Meier U, Zeilinger FS. Der Normaldruckhydrozephalus. Berlin: PVV Science Publications, 2000.

14 Raftopolous C, Chaskis C, Delecluse F, et al. Morphological quantitative analysis of intracranial pressure waves in normal pressure hydrocephalus. Neurol Res 1992;14:389-96.

15 Hebb AO, Cusimano MD. Idiopathic normal pressure hydrocephalus: a systematic review of diagnosis and outcome. Neurosurgery 2001;49:1166-84.

16 Greitz T. Effect of brain distension on cerebral circulation. Lancet 1969;26:863-5.

17 Meyer JS, Tachibana H, Hardenberg JP, et al. Normal pressure hydrocephalus: influences on cerebral hemodynamic and cerebrospinal fluid pressure-chemical autoregulation. Surg Neurol 1984:21:195-203.

18 Kimura M, Tanaka A, Yoshinaga S. Significance of periventricular hemodynamics in normal pressure hydrocephalus. Neurosurgery 1992;30:701-5.

19 Tanaka K, Kimura M, Nakayama Y, et al. Cerebral blood flow and autoregulation in normal pressure hydrocephalus. Neurosurgery 1997:40:1161-7. 
20 Waldemar G, Schmidt JF, Delecluse F, et al. High resolution SPECT with (99mTc)-d, I-HMPAO in normal pressure hydrocephalus before and after shunt operation. J Neurol Neurosurg Psychiatry 1993;56:655-64.

21 Mori K, Maeda M, Asegawa S, et al. Quantitative local cerebral blood flow change after cerebrospinal fluid removal in patients with normal pressure hydrocephalus measured by a double injection method with N-isopropyl-p-[(123)!] iodoamphetamine. Acta Neurochir 2002;144:255-63

22 Black P McL, Ojeman RG, Tzouras A. CSF shunts for dementia incontinence, and gait disturbance. Clin Neurosurg 1985;32:632-51.

23 Liu G, Sobering G, Duyn J, et al. A functional MRI technique combining principles of echo-shifting with a train of observations (PRESTO). Magn Reson Med 1993;30:764-8.

24 Miethke C, Affeld K. A new valve for thet treatment of hydrocephalus. Biomed Tech 1994:39:181-7.

25 Bortz J, Lienert GA, Boehnke K. Verteilungsfreie Methoden in der Biostatistik. Berlin: Springer-Verlag, 2000.

26 Kristensen B, Malm J, Fagerlund M, et al. Regional cerebral blood flow, white matter abnormalities and cerebrospinalö luid hydrodynamics in patients with idiopathic adult hydrocephalus syndrome. J Neurol Neurosurg Psychiatry 1996;60:282-8.
27 Larsson A, Bergh AC, Bilting M, et al. Regional cerebral blood flow in normal pressure hydrocephalus: diagnostic and prognostic aspects. Eur J Nucl Med 1994;21:118-23.

28 Tamaki N, Kusunoki T, Wakabayashi T, et al. Cerebral hemodynamics in normal pressure hydrocephalus. Evaluation by $131 \mathrm{Xe}$ inhalation method and dynamic CT study. J Neurosurg 1984;61:510-14.

29 Del Bigio $M$, Bruni E. Changes in periventricular vasculature of rabbit brain following induction of hydrocephalus and after shunting. $J$ Neurosurg 1988;69:115-20.

30 Kushner M, Younkin D, Weinberger J, et al. Cerebral hemodynamics in the diagnosis of normal pressure hydrocephalus. Neurology 1984;34:96-9.

31 Mamo HL, Meric PhC, Ponsin JC, et al. Cerebral blood flow in normal pressure hydrocephalus. Stroke 1987;18:1074-80.

32 Fisher CM. Hydrocephalus as a cause of disturbances of gait in the elderly. Neurology 1982;32:1358-63.

33 Zimmer R, Leucht S, Radler T, et al. Variability of cerebral blood flow deficits in $99 \mathrm{mTc}-\mathrm{HMPAO}$ SPECT in patients with Alzheimer's disease. $J$ Neural Transm 1997;104:689-701.

NEURONLINE

\section{Amoebaweb: www.vanguard.edu/faculty/ddegelman/amoebaweb Classics in the History of Psychology: http://psychclassics.yorku.ca}

A good web directory is a valuable site that can lead you to places even "Google" may not take you. Amoebaweb must be commended as a vast directory of psychology links, maintained by Douglas Degelman, a professor of psychology at Vanguard University of Southern California. It has a simple, no frills interface, is mercifully free of advertising, covers a huge range of topics within psychology, psychiatry, and neuropsychology, and is well worth a look.

It led me to Classics in the History of Psychology, where full text versions of articles by Broca, Darwin, Freud, Galton, and Janet are available. Whole books, for example William James' The principles of psychology, are also here and because they are digital can be searched by any textword. A search using the word "volition", for example, obtained 95 hits among the articles available.

It would be satisfying to see a similar website devoted to neurology and neurosurgery articles that are now past their copyright date. The internet has the ability to resurrect old scientific material that currently lies out of sightin particular books and journals written before Medline's 1966 cut off. Some encouraging steps forward in this direction have been made by Oldmedline (http://www.nlm.nih.gov/ databases/databases_oldmedline.html),

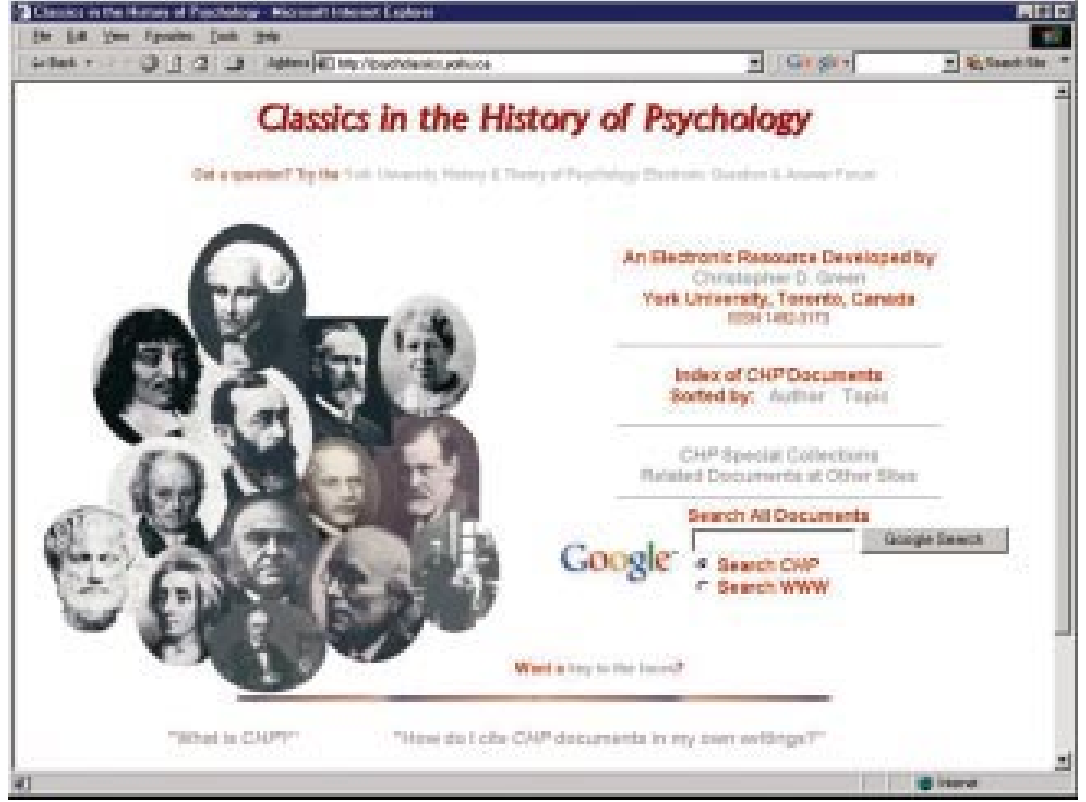

which covers journal articles from 19581965, PsycINFO (http://www.apa.org/ psycinfo), which has abstracts of some articles going back to 1887 and some books to 1938, and the 100 years of Brain archive (http://brain.oupjournals.org), which has a searchable table of contents (but no abstracts until 1981) of all issues of Brain going back to the first in 1878 .

J Stone

Research Fellow in Neurology, Department of Clinical Neurosciences, University of Edinburgh, Western General Hospital, Crewe Road, Edinburgh EH4 2XU; email: jstone@skull.dcn.ed.ac.uk 